\section{IN BRIEF}

\section{$\Rightarrow$ PAEDIATRIC RHEUMATOLOGY}

\section{Long-term safety of canakinumab in systemic JIA}

In a long-term extension of two phase III studies assessing the safety and efficacy of canakinumab (a fully human anti-IL-1 $\beta$ monoclonal antibody) in patients with active systemic juvenile idiopathic arthritis (JIA), treatment efficacy at 6 months was maintained for up to 5 years. No new safety findings were reported and treatment was associated with glucocorticoid discontinuation or substantial reduction in glucocorticoid dose. Overall, 102 patients (58\%) discontinued canakinumab, mainly because of treatment inefficacy. A higher rate of discontinuation was noted for late responders than for early responders ( $81 \%$ versus $29 \%$ ), indicating that early response is a predictive factor of long-term outcome.

ORIGINAL ARTICLE Ruperto, N. et al. Canakinumab in patients with systemic juvenile idiopathic arthritis and active systemic features: results from the 5 -year long-term extension of the phase III pivotal trials. Ann. Rheum. Dis. https://doi.org/10.1136/ annrheumdis-2018-213150 (2018)

\section{$\Rightarrow$ SYSTEMIC LUPUS ERYTHEMATOSUS}

\section{Ustekinumab - a novel treatment?}

The addition of ustekinumab, an IL-12 and IL-23 inhibitor, to standard-of-care treatment was more efficacious than placebo in the treatment of patients with active systemic lupus erythematosus (SLE) in a double-blind phase II randomized controlled trial. More patients in the ustekinumab group achieved an SLE responder index-4 (SRI-4) response at week 24 than in the placebo group $(37(62 \%)$ versus $14(33 \%) ; P=0.006)$. The safety profile of ustekinumab was consistent with that reported in previous trials of ustekinumab for other diseases.

ORIGINAL ARTICLE van Vollenhoven, R. F. et al. Efficacy and safety of ustekinumab, an IL-12 and IL-23 inhibitor, in patients with active systemic lupus erythematosus: results of a multicentre, double-blind, phase 2, randomised, controlled study. Lancet. https://doi.org/10.1016/S0140-6736(18)32167-6(2018)

\section{$\Rightarrow$ GOUT}

\section{IL-1 $\beta$ blockade prevents gout attacks}

In a post hoc analysis of data from CANTOS, a large double-blind placebo-controlled trial of canakinumab treatment for patients with established atherosclerotic disease, canakinumab treatment was associated with a $\sim 50 \%$ reduction in risk of a first gout attack, but not with a change in serum uric acid levels. The reduction in risk of incidence gout was observed for all baseline concentrations of serum uric acid, suggesting that IL- $1 \beta$ blockade can prevent gout attacks independent of serum uric acid levels. ORIGINAL ARTICLE Solomon, D. H. et al. Relationship of interleukin- $1 \beta$ blockade with incident gout and serum uric acid levels: exploratory analysis of a randomized controlled trial. Ann. Intern. Med. https://doi.org/10.7326/M18-1167 (2018)

\section{$\Rightarrow$ RHEUMATOID ARTHRITIS}

\section{DMARD-related adverse events lower persistence}

In a study of patients with methotrexate-refractory rheumatoid arthritis, the addition of sulfasalazine and hydroxychloroquine to methotrexate (triple therapy; $n=171$ ) resulted in a lower persistence rate (defined as treatment without a $\geq 90$-day gap in therapy) than the addition of a TNF inhibitor $(n=2,125)$. Over a 12 month period, $45 \%$ of patients in the TNF inhibitor group persisted with treatment, compared with $18 \%$ of patients in the triple therapy group. Treatment discontinuation was most often because of sulfasalazine-related adverse drug events.

ORIGINAL ARTICLE Erhardt, D. P. et al. Low persistence rates in rheumatoid arthritis patients treated with triple therapy are attributed to adverse drug events associated with sulfasalazine. Arthritis Care Res. https://doi.org/10.1002/acr.23759 (2018)

\title{
Nanoparticles in neutrophil clothing
}

Neutrophils are key immune cells in rheumatoid arthritis (RA) that are known for their involvement in perpetuating, but also in resolving, inflammation. One mechanism used by neutrophils to control inflammation is the production of natural vesicles (such as exosomes and microparticles), which have been shown to be joint-protective in mouse models of RA.

In a new study published in Nature Nanotechnology, researchers show how semisynthetic nanoparticles (NPs) coated in neutrophil cell membrane can also have joint-protective effects in mouse models of RA. "We collected the plasma membrane of activated neutrophil cells and then coated the membrane onto a synthetic nanoscale particle core, forming neutrophil-membrane-coated nanoparticles (neutrophil-NPs)," states corresponding author Liangfang Zhang. "The resulting neutrophil-NPs were then applied locally to inflamed joints."

Intra-articular injection of neutrophil-NPs reduced disease severity and joint damage in two mouse models of RA (collagen-induced arthritis and a transgenic model of inflammatory arthritis) to a level comparable with mice treated with anti-TNF or anti-IL- $1 \beta$ antibodies.

"Neutrophil-NPs were able to effectively interact with and absorb various types of inflammatory cytokines that would otherwise have interacted with real neutrophils," says Zhang.

The researchers also investigated the ability of neutrophil-NPs to penetrate cartilage. Fluorescently labelled neutrophil-NPs could be detected to a depth of $140 \mu \mathrm{m}$ from the cartilage surface in explants, whereas erythrocyte-membranecoated NPs could only be detected to a depth of $30 \mu \mathrm{m}$. Neutrophil-NPs were also seen in close proximity to chondrocyte nuclei, indicating that the NPs might have been taken up by the chondrocytes. In explants

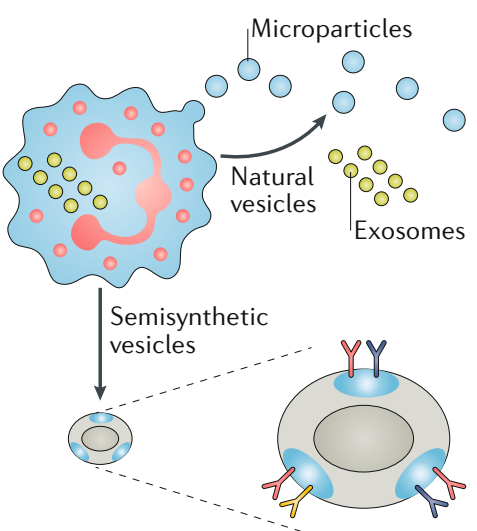

Credit: Adapted from Thomas, B.L. \& Perretti, M. Neutrophil wrap. Nat. Nanotechnol. https://doi. org/10.1038/s41565-018-0260-6 (2018)

treated with IL-1 $\beta$, neutrophil-NPs reduced cartilage damage by removing IL- $1 \beta$ from the environment.

"The development of therapeutic approaches based on the modification of NPs with cell membrane represents an important leap forward in the field of nanomedicine since they combine the benefits of both human-made and natural materials," explains Massimo Bottini, who was not involved in this study. "Although the authors did not assess the mechanism of interaction between neutrophil-NPs and chondrocytes, the ability of neutrophil-NPs to enter the cartilage and target the chondrocytes is an important finding," he continues. "The next step should be to test the therapeutic efficacy of systemically administered neutrophil-NPs in mouse models of arthritis as both a therapeutic agent and a chondrocyte-specific drug delivery system."

"We are very interested in moving this technology forward to evaluate its clinical application potential; however, a major challenge that we have to overcome is the large-scale manufacturing of the neutrophil-NPs to GMP quality," concludes Zhang.

\section{Joanna Collison}

ORIGINAL ARTICLE Zhang, Q. et al. Neutrophil membrane-coated nanoparticles inhibit synovial inflammation and alleviate joint damage in inflammatory arthritis. Nat. Nanotechnol. https: // doi.org/10.1038/s41565-018-0254-4 (2018) 